\title{
IRJAR
}

Interdisciplinary Research

Journal \&Archives

IRJAR/JRIA

Article

\section{Covid-19 dans la ville de Kinshasa : Représentations sociales chez les fugitifs du confinement.}

\author{
Mabakutuvangilanga Ntela Simon-Decap, RN PhD ${ }^{1,2 *}$; Feti Kisiata Julien, MScN ${ }^{3,4 *}$; Mukeba \\ Nkashama Jacques, MScN² ; Munanga Kabasele Aimé, $\mathrm{MScN}^{2}$; Ramazani Jean-Bosco, $\mathrm{MD}^{5}$; Ramazani \\ Bin Eradi Imani, $\mathrm{MScN}^{5}$; Matoko Nzinga Flavienne, $\mathrm{RN}^{6}$; Margat Aurore, $\mathrm{PhD}^{7}$; Rothan-Tondeur \\ Monique, $\mathrm{RN}, \mathrm{PhD}^{7,8}$
}

\begin{abstract}
${ }^{1}$ Université Sorbonne Paris Nord, Chaire Recherche Sciences infirmières, Laboratoire Éducations et Pratiques de Santé (LEPS), (UR 3412), UFR SMBH, F-93017, Bobigny, France ;

${ }^{2}$ Institut Supérieur des Techniques Médicales de Kinshasa, République Démocratique du Congo ;

${ }^{3}$ Institut Supérieur des Techniques Médicales de Kikwit, République Démocratique du Congo ;

${ }^{4}$ Centre de Recherche en Sciences Humaines, Kinshasa, République Démocratique du Congo ;

${ }^{5}$ Institut Supérieur des Techniques Médicales de Kindu, ville de Kindu, République Démocratique du Congo ;

${ }^{6}$ Programme National de l'Hygiène des Frontières, République Démocratique du Congo ;

${ }^{7}$ Assistance Publique - Hôpitaux des paris (AP HP) Chaire recherche Sciences Infirmières Paris France ;

8 Université Sorbonne Paris Nord, Chaire Recherche Sciences infirmières, Laboratoire Éducations et Pratiques de Santé (LEPS), (UR 3412), UFR SMBH, F-93017, Bobigny, France.
\end{abstract}

* Auteur correspondant : julienfeti@gmail.com

Reçu : ; accepté : ; publié :

\section{Résumé}

À l'annonce du verrouillage de Kinshasa (la capitale de la République démocratique du Congo) en raison de l'augmentation des cas d'infections à coronavirus, une vague de peur et d'inquiétude a surgi parmi la population de la ville, provoquant un déplacement massif de la population vers les provinces voisines. Il s'agit de contextualiser une étude phénoménologique qualitative explorant la représentation sociale du COVID-19, les motivations influençant le déplacement de population et les discours des sujets sur les stratégies de soins ou « artefacts thérapeutiques» proposés par cette population de Kinshasa. L'analyse de 19 entretiens semi-structurés a mis en évidence la présence de cinq catégories de représentations du COVID-19: maladie imaginaire, maladie des hommes d'affaires, invention à des fins démographiques, guerre entre États et châtiment divin. En outre, quatre types de motivation ont influencé les mouvements de la population : crise socio-économique, insécurité, interdiction des églises et, accès facile aux traitements traditionnels. Cette étude montre enfin que la population fugitive utilise des thérapies traditionnelles (herboristes et croyances traditionnelles, y compris la prière et la sorcellerie) pour faire face à cette pandémie. Une amélioration des connaissances, un renforcement du système communicationnel et des interventions visant le changement des représentations sociales à l'origine des images négatives en matière de COVID-19 sont conseillés.

\footnotetext{
Abstract

Upon the announcement of lockdown of Kinshasa (The capital city of the Democratic Republic of Congo) due to increase cases of corona virus infections, a wave of fear and concern arose among the population of the city
} 
causing a massive population's displacement towards neighboring provinces. This is to contextualize a phenomenological qualitative study exploring the social representation of COVID-19, the motivations influencing the shift of population and the discourses of the subjects on the care strategies, or "therapeutic artifacts" proposed by this population of Kinshasa. The analysis of 19 semi-structured interviews highlighted the presence of five categories of representations of COVID-19: imaginary disease, disease for businessmen, invention for demographic purposes, war between states and, divine punishment. In addition, four types of motivation have influenced the movement of the population: socioeconomic crisis, insecurity, ban of churches and, easy access to traditional treatment. This study finally shows that the fugitive population uses traditional therapies (herbalists and traditional beliefs, including prayers and sorcery) to cope with this pandemic. Improving knowledge, strengthening the communication system and interventions aimed at changing social representations causing negative images of COVID-19 are recommended.

Mots clés : COVID-19, Représentations sociales, Artefacts thérapeutiques, Confinement Keywords: COVID-19, Social representations, Therapeutic artifacts, Lockdown

\section{Introduction}

L'infection à coronavirus (COVID-19) est l'événement sanitaire important qui a marqué la fin de l'année 2019 et sans doute fait et fera parler de l'année $2020^{\{1\}}$. D'abord, circonscrite au départ à la chine, cette infection a vite gagné la planète, faisant d'elle parmi les infections les plus dévastatrices que la planète n'ait jamais connue ${ }^{22\}}$. «Après sa découverte à la fin 2019 chez de nombreux patients souffrant de pneumonie à Wuhan (province de Hubei, Chine), la propagation planétaire de ce virus tient le monde entier en haleine ${ }^{[3]}$. Le nouveau coronavirus ( $\mathrm{CoV})$ est désormais appelé " SARS-CoV-2 ». Il est à l'origine de la «COVID19 » soit « the corona virus disease-19 » correspond à l'année de sa découverte $\aleph^{\{4\}}$. La propagation rapide de cette épidémie a créé dans toutes les populations des sentiments de panique, de désolation et de grandes inquiétudes. Déclarée par l'OMS comme pandémie le 11 mars 2020, la COVID-19 était vite considérée comme une urgence de santé publique ${ }^{\{5}$. Cette époque chaotique et irréelle que traverse le monde affecte le système de santé, d'autant plus que celui-ci est précaire, comme c'est le cas des pays d'Afrique ${ }^{[6]}$.

La République Démocratique du Congo (RDC), encore menacée par la grande épidémie à virus Ebola, se voit contrainte à gérer la COVID-19. Les mesures de prévention de cette pandémie (confinement, lavage des mains, restriction des salutations et des accolades, fermeture des lieux d'amusement, la fermeture des frontières, la fermeture des Églises...) suscitent des bouleversements sociétaux [7]. La solidarité constatée dans la culture congolaise se voit brisée par ces mesures pourtant bénéfiques, imposant aux populations un autre rythme de vie au nombre de personnes infectées par le nouveau coronavirus, qui augmente rapidement dans le monde.

Le confinement annoncé, du samedi 28 au mardi 31 mars 2020 inclus, dans la ville de Kinshasa est une mesure qui consiste au respect strict des consignes de sécurité afin d'éviter le plus possible les déplacements et les rencontres. Dans ce contexte, deux raisons ont motivé ce confinement: limiter les contaminations et éviter la propagation de l'épidémie dans les provinces non touchées. En ce sens et pour le bien de tous les habitants de la ville de Kinshasa, toute autre activité que le strict nécessaire est proscrit ${ }^{16}$. Bien que le confinement soit une mesure importante pouvant réduire le nombre de malades et de morts, il a été annoncé dans la ville de Kinshasa alors que la population n'y avait pas été préparée. Une vague de peur et d'inquiétude a surgi dans la société, provoquant ainsi un déplacement massif des populations vers les provinces voisines (Kwilu et Kongo-central).

\section{Cadre conceptuel et objectifs de l'étude}

Cette étude s'appuie sur la théorie des représentations sociales de Serge Moscovici ${ }^{88}$. 
Élaborée en psychologie sociale, elle est appréhendée ici comme « un ensemble d'informations, de croyances, d'opinions et d'attitudes à propos de la COVID 19 ». Le choix de cette théorie privilégiant la pensée naturelle plutôt que scientifique a été motivé par le fait qu'elle prend en compte l'aspect d'« étrangeté de l'objet (COVID-19) pour la population », la « carence informationnelle » et la "menace de l'identité collective » qu'elle suscite. Baser les analyses sur une représentation, c'est: " observer comment cet ensemble de valeurs, de normes sociales et de modèles culturels sont pensés et/ou vécus par des individus d'une société ${ }^{19}$. C'est aussi étudier comment s'élabore, se structure logiquement, et psychologiquement, l'image de ces objets sociaux ». En d'autres termes, il s'agit spécifiquement ici de comprendre comment les sujets se représentent-ils la COVID-19, $\mathrm{d}$ 'analyser la manière dont se construisent les connaissances sur le sujet et la manière dont circulent les savoirs sur son traitement. Ainsi, devant une infection à coronavirus mal connue par la population étudiée, il se crée dans la population de la ville de Kinshasa des idées nouvelles qui influencent l'adoption d'un comportement nouveau. La COVID-19 en général et particulièrement le confinement considéré comme situation étrangère dans la société, les individus ou les groupes réagissent et construisent progressivement un savoir inattendu et généralement non objectif.

L'objectif de cette étude est celui de comprendre les représentations de l'infection à COVID-19 par les populations de Kinshasa fuyant le confinement dans la ville.

\section{Approche méthodologique}

\subsection{Contexte de l'étude}

Cette étude s'est déroulée dans la ville province de Kinshasa spécifiquement au quartier de Mitendi à la sortie de la province du Congo Centrale pour entrer dans la province du Kongo central. La province de Kinshasa, capitale de la République Démocratique du Congo, est une ville peuplée de plus de 10 millions d'habitants ${ }^{\{10\}}$. Il s'agit d'une ville cosmopolite avec une population-accordéon, fluctuant chaque année à cause de l'attraction qu'elle exerce en tant que grande ville où sont concentrées les instances politicoadministratives, les grandes universités, les grands marchés, etc. Kinshasa occupe une boucle dans le fleuve Congo, une plaine alluviale bordée de collines sablonneuses sur lesquelles tout peuplement humain reste précaire en raison de la tendance érosive du territoire. Sept pour cent seulement des chefs de famille sont nés à Kinshasa; le reste vient des provinces dont 60\% directement de la campagne ${ }^{[11]}$. Par ailleurs, le rôle complexe et souvent ambivalent que de nombreux chefs de terres autochtones continuent de tenir dans le processus d'expansion urbaine perdure à Kinshasa ${ }^{\{2\}}$. Cette ville a connu d'importantes transformations économiques, sociales et démographiques au cours ces dernières décennies ${ }^{[10\}}$. Malgré ce développement fulgurant, les infrastructures nécessaires à l'hygiène et à l'assainissement (distribution de l'eau potable, évacuation des eaux usées et des déchets) ne sont pas suffisamment développées [12\}. C'est dans ces conditions d'hygiène extrêmement précaires que les cas d'infection à coronavirus ont été déclarés dans le pays, dont Kinshasa a été l'épicentre ${ }^{\text {13\}. }}$.

Le choix de ce site de Mitendi pour l'étude est motivé par le fait que ce quartier est situé à la sortie de la capitale Kinshasa vers la province $\mathrm{du}$ Bas-Congo, où était érigée la barrière circonstancielle nécessaire pour un contrôle systématique des populations fuyant la ville de Kinshasa, à l'annonce du confinement au COVID-19 et ceci, malgré l'existence de différents services (le programme national de l'hygiène aux frontières (PNHF), l'Agence nationale de renseignement, la police nationale...).

\subsection{Type d'étude}

Considérant les objectifs de l'étude et le cadre théorique privilégié, nous optons pour une étude qualitative phénoménologique ${ }^{\{14\}}$.

\subsection{Méthode d'échantillonnage et sélection des participants}

Un modèle d'échantillonnage non probabiliste a été privilégié. De ce fait, un choix raisonné des sujets se présentant avant le poste de frontière entre la ville de Kinshasa et la province du Kongo central a été réalisé. Il s'agit ici d'un 
choix dont la sélection des sujets tient compte des désirs ou des opinions du responsable de l'étude. L'interview était donc réalisée avant la barrière afin d'éviter le biais lié au stress émotionnel du sujet au regard des tracasseries de différentes barrières policières qui imposent un contrôle physique et la peur d'y être retenu pour une éventuelle fièvre. Il s'agit d'un poste de contrôle circonstanciel mis en place à l'annonce du confinement dans le souci d'empêcher la propagation de l'infection à COVID-19 de la ville de Kinshasa vers la province du Kongo central, puis au contrôle sanitaire (notamment du service du programme national de l'hygiène des frontières) qui interroge la personne sur les antécédents d'avoir vécu dans un contexte à forte incidence de COVID-19. De plus, une prise de la température est réalisée. Toutes les personnes avec fièvre persistante étaient mises en observation et acheminées vers l'Institut National de Recherches Biomédicales (INRB) pour examen approfondi. Dans le cas contraire, la personne est libérée. Ainsi, les sujets sélectionnés pour l'étude devaient vivre dans la ville de Kinshasa, fuir le confinement dans le cadre de la COVID-19 et se diriger vers la province du Kongo Central en empruntant le national numéro 1 (Route de Matadi). Un autre critère d'inclusion était l'acceptation du sujet à participer à l'étude. Tous les passagers quittant la ville de Kinshasa vers cette province pour une autre raison (commerçant, chauffeurs des poids-lourds, voyage pour des situations de deuil...) n'ont pas été inclus. La taille de l'échantillon a été déterminée sur base de la saturation des résultats ${ }^{\{15\}}$. Cette saturation a été atteinte lorsqu'aucune nouvelle information n'enrichissait encore les données de notre étude.

\subsection{Collecte des données}

Des entretiens semi-directifs ont été réalisés chez les fugitifs ayant passé au poste de contrôle du service du programme national de l'hygiène des frontières. De ce fait, un guide d'entretien contenant trois grandes questions se rapportant aux dimensions développées dans la théorie des représentations sociales était utilisé. La première était : Comment vous représentez-vous la COVID-19? Il s'agit d'une question en rapport avec les informations que possèdent les sujets sur la COVID-19. La deuxième question est en rapport avec leur croyance sur le confinement et leurs attentes sur la fuite préconisée «qu'est-ce qui aurait motivé votre déplacement vers la province du Kongo central à l'annonce du confinement ? ». C'est-à-dire la prise de position sur leur déplacement de la ville de Kinshasa vers les provinces voisines. La dernière question tenait compte des actions thérapeutiques visant à lutter contre le COVID-19: "comment comptez-vous vous prévenir contre cette maladie? » Elle s'est appuyée sur l'influence sociétale ou environnementale ou encore sur leurs attitudes sur le choix du traitement envisagé pour prévenir ou guérir la COVID-19. Les entretiens ont été enregistrés en français et en 2 langues locales (le Kikongo et le Lingala).

\subsection{Analyse des données}

Après chaque entretien, une retranscription sur outil informatique a été réalisée par l'enquêteur. Ensuite, la traduction des entretiens réalisée en langues locales vers le Français a été réalisée par deux personnes indépendantes ayant la maîtrise de ces trois langues reliées à l'étude (Français comme langue officielle et Kikongo, Lingala qui sont des langues locales). De ce fait, chaque traducteur a d'abord travaillé de manière indépendante et une comparaison de tous les discours traduits était réalisée. Toutefois, le recours à la troisième personne ayant les mêmes caractéristiques que les traducteurs précédant a été nécessaire dans le souci de départager les éléments dont les points de vue étaient divergents.

Après l'étape de la traduction, une analyse du contenu a été réalisée. Elle s'est inspirée de grandes étapes, énoncées par L'Écuyer comme une démarche en cinq étapes : la collecte des données verbales; la lecture des données; la division des données en unités; l'organisation et l'expression des données brutes dans le langage de la discipline, et la synthèse des résultats ${ }^{\{16\}}$. Ainsi partant du thème principal, des sous-thèmes ont été dégagés, lesquels sousthèmes ont aussi dégagé différentes catégories et des verbatim. Les données ont été non seulement soumises aux trois chercheurs 
indépendants pour leur validité mais aussi à la vérification auprès des participants à l'étude.

\subsection{Aspect éthique}

L'étude a été menée dans un contexte de crise sanitaire, au moment du confinement de la ville de Kinshasa. La recherche a cependant été conduite de façon à suivre les principes éthiques habituellement appliqués en pareilles circonstances. Ainsi, le consentement des personnes s'est fait par leur participation libre et éclairée à l'entretien. Les investigateurs se sont assurés de la bonne compréhension des informations délivrées et le consentement de chaque sujet interrogé a été systématiquement recueilli, tout comme l'autorisation verbale des autorités du poste de contrôle de Mitendi. De même, le caractère confidentiel de données enregistrées ainsi que l'anonymat a été pris en compte: l'utilisation des données recueillies était sous le contrôle exclusif de l'investigateur principal. Aucune donnée recueillie ne permettait d'identifier les participants.

\section{Résultats}

\subsection{Caractéristiques des répondants}

Un total de 15 sujets en majorité de sexe masculin dont l'âge variait entre 22 ans et 67 ans, ayant pour la plupart un niveau d'études secondaire avec un faible niveau socioéconomique et vivant des travaux champêtres (comme activité principale) ont été interrogés.

\subsection{Résultats relatifs à la représentation sur la COVID-19}

Les résultats ont été groupés en trois (3) sous thèmes qui sont : la signification traditionnelle accordée de la COVID-19, les motivations influençant le déplacement de la population à l'annonce du confinement et les artefacts thérapeutiques.

\subsubsection{Signification traditionnelle accordée à la COVID-19}

Maladie psychologique

La plupart de personnes interviewées doutent de l'existence réelle de la pandémie à COVID19 en République Démocratique du Congo et croient à une invention occidentale spécifiquement pour un but démographique.

" ehhhh cette maladie n'existe pas, elle est imaginaire...ils veulent réduire la population mondiale»; "...Pour moi, cette maladie n'existe pas réellement. Si elle existe chez nous, qu'on nous montre les personnes qui en souffrent. Nous comprenons que les mindeles (les blancs) ou les occidentaux veulent envahir notre terre, voilà pourquoi...»; "moi je doute de cette maladie. Ces gens-là sont capables d'exterminer toute l'Afrique pour qu'ils occupent notre terre. Je rentre chez moi...»; "Croyez-vous réellement à cette maladie? Qu'on parle de cela en Europe, aux ÉtatsUnis, en Chine mais pas chez nous. C'est une invention occidentale... ». «La COVID-19 n'existe que dans leur tête, en n'est vraie en réalité...car les morts ne sont pas visibles... ».

Une manipulation des hommes politiques

En dehors des représentations quant à l'existence de la COVID-19, les personnes interrogées ont également souligné dans leurs discours une possible manipulation $\mathrm{du}$ gouvernement.

"uhhh, Ce sont les grandes puissances qui se poignardent et nous nous devons endurer en bloquant les actions dans la ville... »; "Nous savons que c'est politique cette histoire de COVID. On en a marre avec cette affaire»; "Ne me parle pas surtout de ce COVID-là, moi j'ai plus peur du confinement qui me fait fuir, car cette histoire est inventée par des politiciens"; "tu ne vois pas ça touche plus les grandes personnes? Et c'est nous le petit peuple qui subissons...».

\section{Occasion pour se faire de l'argent}

Un grand nombre des sujets interrogés considèrent que la COVID-19 est une maladie des personnes qui font des affaires.

" uhhhhh, le corona est une maladie des riches. Comment expliquer qu'elle soit ramenée chez nous par des voyageurs? Et maintenant c'est nous qui subissons et on 
s'organise pour que nous restions bloqués chez nous...»; "moi je pense qu'en réalité nous ne sommes pas concernés par cette maladie. Je n'ai pas voyagé vers l'Europe, comment serai-je contaminé...?»; "Comment expliquer que ce sont les personnes de la classe sociale élevée (Homme politique, professeurs, riches...qui en meurent...)?»

Conflits des grandes puissances

Les sujets affirment que la survenue de la COVID-19 est corollaire à des conflits entre les grandes puissances et ne concernent donc pas l'Afrique.

"Ce sont les conflits entre les grandes puissances qui en sont la cause et nous on subit chez nous ici... »; "C'est la recherche $d u$ leadership entre les grandes puissances notamment les États-Unis, la chine, la France...qui en est la cause ».

Stratégie de dépeuplement mondial

Des sujets ont allégué que la COVID-19 n'est qu'une invention pour dépeupler le monde spécifiquement pour se débarrasser des personnes âgées qui constituent une charge pour leurs États.

"Ce sont les gouvernements des pays occidentaux qui en ont créé dans le but de réduire les effectifs des personnes âgées qui constituent aujourd'hui une source des dépenses publiques..."; "Moi je pense que cette maladie a été mise en place pour réduire la population mondiale. Mais pas chez nous car...».

Colère divine

Les sujets ont aussi souligné que la COVID-19 serait une extension de la colère de Dieu Ah, mais c'est Dieu qui nous sanctionne car on a commis trop de mal vraiment » $E$; « non Dieu est fatigué de nous, on ne s'aime pas icibas voilà pourquoi il nous crée une confusion pour montrer sa puissance..."; "c'est une interpellation de Dieu pour tous les péchés du monde qui sont en exagération...».

\subsubsection{Motivations influençant le déplacement de la population à l'annonce du confinement}

L'aggravation de la crise socioéconomique

Le refus de se confiner par la population est motivé par la croyance anticipée selon laquelle la COVID-19 aggraverait la crise sociale.

"Mais nous vivons déjà très mal, si on doit accepter se bloquer dans cette ville, que va-til nous arriver? Comment allons-nous mange? »; "se confiner signifie qu'on doit rester chez soi. Or nous vivons ici du jour le jour, je dois vendre pour que ma famille trouve à manger, que faire alors... le mieux c'est de quitter cette ville; Ça chauffe déjà avant le confinement, ce qui arrivera pendant le confinement est donc une grande inconnue (Sic), non, je m'en vais chez moi au village où je peux trouver à manger pour ma famille...».

\section{L'insécurité}

Plusieurs sujets ont justifié la fuite du confinement par crainte de l'insécurité. En effet, l'annonce du confinement de la commune de la Gombe supposait que des criminels de cette commune devraient quitter celle-ci pour les communes périphériques.

"Mais la commune de la Gombe est celle qui héberge les plus des criminels dans cette ville. A l'annonce $d u$ confinement de cette commune, nous avions ou des criminels quitter cette commune vers notre quartier. Non, le mieux c'est de s'en aller au village où ces actions sont rares"; "Mon cher, nous avions vu certains bandits quitter la commune de la Gombe pour notre quartier, ils ont refusé aussi d'être confinés. Ça fait tellement peur de vivre dans l'incertitude, nous allons mes deux enfants, ma femme et moi-même en province... ».

L'angoisse due à la fermeture des églises

Pour plusieurs personnes interviewées, les mesures qui accompagnent le confinement annoncé dans la ville de Kinshasa (fermeture des églises) expose à la condamnation de la population au regard de la place accordée à la prière pour guérir la COVID-19.

"On nous dit paraît-il que cette maladie n'a pas de traitement. Or dans des contextes comme celui-ci, notre force réside sur la prière. Donc je ne saurai pas rencontrer mon 
pasteur...Au village on trouvera des solutions, je pars... "; "La force divine et les prières des intercesseurs doivent jouer leurs rôles dans des pareils cas. Or on ne peut pas prier en groupe, là c'est vouloir nous sacrifier tous...».

A la recherche du traitement traditionnel

Les répondants justifient aussi leur motivation de se déplacer vers la province du Kongo central à l'annonce du confinement de la ville de Kinshasa par la facilité de trouver en milieu rural toutes les plantes traditionnelles pouvant prévenir ou guérir selon eux la COVID-19.

"Allons-y, au village on peut trouver toutes ces plantes efficaces pour lutter contre la COVID-19, mais ici, tout est couteux...»; "le Kongo bololo, le gingembre, toutes les plantes médicinales ont brusquement augmenté de prix à partir du moment où on a parlé de leur efficacité sur la COVID-19. Ça c'est nous tuer, car au village on a tout gratuitement...».

\subsubsection{Artefacts thérapeutiques}

Des thérapeutiques traditionnelles

Plusieurs personnes ont évoqué l'efficacité de l'utilisation des plantes indigènes pour guérir de la COVID-19.

Traitement à base de Vernonia amygdalina (Kongo bololo)

Dans la majorité de cas, ils ont souligné faire confiance au "Vernonia amygdalina» connu localement sous le nom de «Kongo bololo» pour guérir la COVID-19.

"Eeeee si eux là bà (Sic ?) ils n'ont pas encore trouvé de médicaments, nous faisons confiance à plusieurs remèdes traditionnels notamment le «Kongo bololo»; « nous, depuis longtemps nous prenons le Kongo bololo pour nous guérir de plusieurs maladies même leur maladie ici... »; "Chez nous c'est une mince affaire, nous prenons juste des boissons à base de Kongo bololo, et votre COVID-19 serait partie... ».

Fumigation traditionnelle

Des nombreuses personnes ont révélé avoir confiance à la fumigation traditionnelle «le
Kioko en langue kikongo ». Il s'agit d'une solution bouillie, un mélange de plusieurs catégories des feuilles d'arbres, dont la vapeur est inhalée après s'être convenablement couvert de grosses couvertures. Cela est peut être poursuivie pendant cinq bonnes minutes ou lorsque l'eau est devenue froide. Les personnes interrogées se sont exprimées en ce terme :

"Moi je boullie (Sic) juste des feuilles des manguiers, goyavier et tant d'autres. D'habitude je chauffe convenablement cette solution et la marmite chaude est placée entre mes jambes. Je me couvre ensuite de beaucoup de couvertures, dès que j'ai inhalé la vapeur chaude, je transpire, tout est fini... ". "Pour moi à chaque fois que j'ai eu un contact à risque, au retour chez moi, je mets au feu des feuilles d'arbres et je place la marmite chaude entre mes jambes. Généralement sans habits et je me fais couvrir des habits lourds. Après inhalation de la vapeur, eh bien, mes voies respiratoires se libèrent et je suppose éliminer le virus en question...»; "Moi je n'utilise que la fumigation traditionnelle faite sur base des diverses feuilles d'arbres bouillies et je me force d'inhaler sa vapeur chaude et c'est partie, le virus est éliminé...». "Combinaison de la "Vernonia amygdalina» et de la fumigation traditionnelle ».

D'autres par contre ont souligné faire plus confiance à la combinaison de «Vernonia amygdalina» avec la «fumigation» traditionnelle «Kioko». Ils pensent que cette synergie agirait à la fois à titre préventif et curatif contre la COVID-19.

"Chez nous, nous aimons la combinaison de deux produits traditionnels: la prise $d u$ Vernonia amygdalina et la fumigation traditionnelle ; "C'est vraiment efficace pour la prévention et la guérison de la COVID-19'; ...pour avoir plus d'efficacité, nous combinons le Kongo bololo et la fumigation traditionnelle. C'est très fort, très toléré et pose moins des problèmes. »; ... uhhh moi je combine tous ces jours la boisson à base de Vernonia amygdalina le 
Kioko «fumigation». et ça donne des bons résultats...».

\subsubsection{Les croyances traditionnelles}

Prière religieuse

Les répondants doutant de l'existence réelle de la COVID-19 pensent que la prière serait la meilleure solution pour guérir la COVID-19.

"Nous savons que ce fameux coronavirus ne peut résister face la chaleur divine. Seule la prière peut nous sauver.»; "la COVID-19 est une interpellation divine, il a décidé pour nous châtier donc nous devons prier convenablement. Comment cette maladie doit rendre la recherche impuissante, il faut maintenant prier...».

Invocation fétichisme et incantations coutumières

Trois sujets considèrent la voie fétichiste ainsi que les incantations coutumières comme une solution pour guérir la COVID-19. Les personnes interrogées se sont exprimées en ce terme :
"Ohhhh la COVID-19 n'est pas plus puissante que notre "nganga-nkisi» féticheur E3. Si celui-ci échoue, la COVID19 n'échappera pas non plus à la puissance des cérémonies coutumières du village... »E8; "chez nous, les féticheurs et les cérémonies coutumières agissent ensemble pour guérir les maladies compliquées. Si ici à Kinshasa, la COVID-19 prend place, elle ne résistera pas aux forces ancestrales $»$ E17.

\section{Discussion}

\subsection{Choix de la méthode}

Cette étude cherche à comprendre les représentations de l'infection à COVID-19 par les populations Kinoises fuyant le confinement dans la ville de Kinshasa. De ce fait, l'approche phénoménologique adoptée semble être bien adaptée si l'on se tient à Negura qui considère que « dans l'étude des représentations sociales \{17\}, l'on utilise de plus en plus de techniques de recueil des données dites qualitatives ».

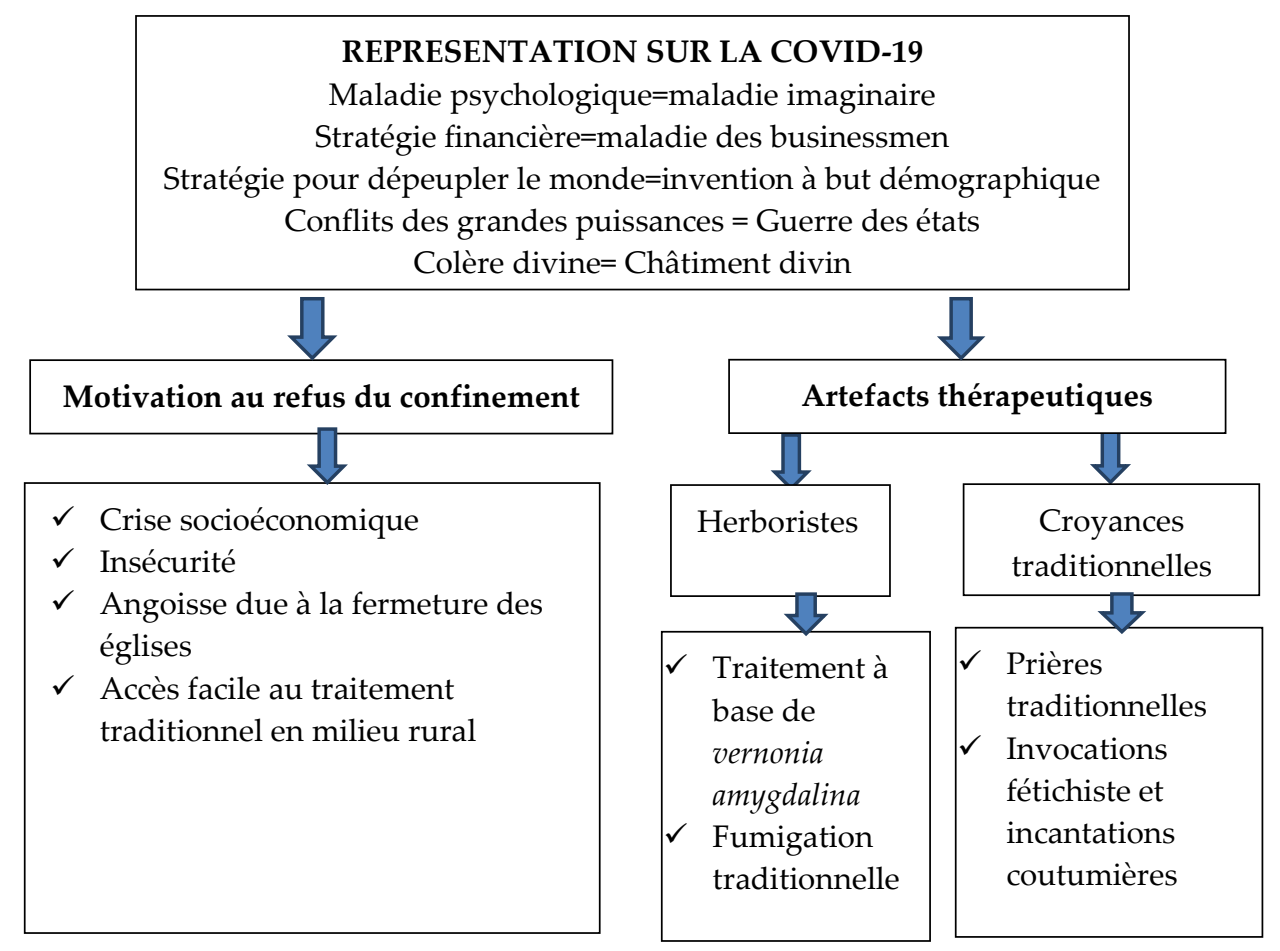

Figure 1: Résumé de l'ensemble des résultats de l'étude

De même, la technique d'analyse de contenu utilisée a permis à travers les entretiens semidirectifs essentiellement dialogiques, « c'est-à- dire que le discours des enquêtés créés dans la communication avec l'enquêteur » de mettre en exergue les réalités représentationnelles qui correspondent à la COVID-19 ${ }^{\{17\}}$. 


\subsection{Principaux résultats}

\subsubsection{Représentation sociales de la COVID-19}

Les résultats de cette étude montrent que la population Kinoise a plusieurs représentations sur cette pandémie. A ce sujet, Valentin Migabo fait remarquer que depuis le 14 février 2020, date à laquelle le premier cas africain de la COVID-19 a été détecté en Égypte, des réactions diverses se sont faits sentir à travers le continent ${ }^{188}$. L'auteur souligne que «des prophéties, des messages véhiculés par certains leaders religieux, y compris des médecins traditionnels et quelques personnalités politiques, ont aussi nourri la conviction que l'Afrique avait des solutions pour faire face à la pandémie »

D'après les analyses des propos recueillis dans cette étude, les principales représentations accordées à la COVID-19 par la population fuyant la ville de Kinshasa à l'annonce du confinement révèlent cinq catégories de significations de COVID-19: une maladie psychologique, donc maladie imaginaire, COVID-19, maladie des hommes d'affaires, COVID 19 : une affaire politiques, COVID-19: une guerre des états, COVID-19: Invention à but démographique). Le schéma ci-dessous illustre les principaux résultats de l'étude.

\section{COVID-19 : maladie imaginaire}

Cette considération n'est pas récente dans le contexte Africain surtout au regard des doutes qui persistent auprès des personnes quant à son existence réelle ou non, surtout en l'absence visible des patients atteints. Cette difficulté a déjà été rencontrée par Hamon-Valanchon lorsqu'il confirme qu'une maladie imaginaire renvoie à un « langage suggestif, indirect, riche de plusieurs sens et ouvert, symbolique et mythique ${ }^{\{19\}\rangle}$. Cette considération selon Hamon-Valanchon dépend sans nul doute, des capacités sociales, culturelles, psychiques des personnes à intégrer cet événement dans leur vie ${ }^{199}$. Moulin en décryptant l'imaginaire à partir des représentations sociales a montré qu'une maladie peut générer un authentique " univers culturel qui peut révéler et questionner les mentalités et les conduites collectives", la maladie étant une forme de construction sociale ${ }^{\{20}$. Cela est lié aux croyances et aux valeurs sociales que peut refléter chaque connaissance scientifique. La considération de la COVID-19, comme une maladie imaginaire, peut ici faire référence à une construction d'une série d'images et de représentations irréelles influencées par la culture. Il y a lieu de réaffirmer les propos d'Augustin Voegele que «les gens bien portants sont des malades qui s'ignorent $»^{\{21\}}$. Par ailleurs, en considérant la COVID-19 comme maladie imaginaire, la population Kinoise s'attache d'abord à l'expérience singulière relative au confinement, au bouleversement de tous les rythmes et rites traditionnels qu'elle suscite. D'autre part, il peut s'agir de la transformation profonde $y$ compris sensorielle du rapport contextuel

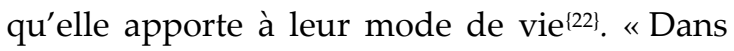
des sociétés où le contact social constitue une norme culturelle omniprésente dans les faits et gestes quotidiens (salutations, repas en famille, réjouissances, pratiques religieuses, etc.), les mesures de distanciation peinent à entrer dans les habitudes malgré les moyens déployés dans la sensibilisation des populations »\{23\}. Par maladie imaginaire, la COVID-19 prend ici un sens d'une maladie qui est dans une large mesure inexistante. Ce qui est mis en jeu ici est l'assimilation du psychogène à l'imaginaire ${ }^{[24\}}$.

\section{COVID-19: Invention à but démographique}

La population interviewée souligne que la déclaration de la COVID-19 avait le but de dépeupler le monde. Cette conception relevée dans les pensées de Kinois est aussi très ancienne. Dumon considère la mortalité comme la première conséquence démographique visible d'une épidémie ${ }^{25\}}$. Depuis longtemps, les grandes épidémies ont dévasté des familles entières abandonnant des terres entières. L'ampleur de la COVID-19 peut se comparer à la capacité létale d'autres épidémies qui ont eu des effets sur la démographie. A ce sujet, l'auteur explicite certaines expériences des épidémies qui ont eu des effets sur le dépeuplement ${ }^{[25]}$. C'est le cas "d'Hippocrate qui parle d'une épidémie grippale à Athènes. En 510 à Rome, une autre épidémie de grippe fit périr 9000 personnes 
dans la ville et dépeupla plusieurs pays d'Europe. Dans ce même continent, la peste noire commencée en 1347, fit de l'ordre de 20 millions de morts, soit un quart de la population pour la seule Angleterre »266. Si en ce temps-là Alfred Sauvy considérait la peste comme l'une des épidémies la plus dévastatrice au fil de l'histoire, aujourd'hui il inclurait sans doute la COVID-19:277. Le dépeuplement dont parle la population fugitive du confinement de la ville de Kinshasa fait allusion, selon elle, à une stratégie mise en place pour exterminer la population Africaine. De ce fait, nous sommes d'avis, qu'il faille intensifier la communication pour faire face à toutes ces considérations erronées qui freinent la lutte engagée contre cette pandémie.

\section{COVID-19 : Maladie des hommes d'affaires}

Alors que la COVID-19 continue de se propager à travers le monde en général et particulièrement en Afrique, des mobilisations des fonds se font sentiri27). Au regard de la conjoncture économique nationale et internationale incertaine dans laquelle survient la COVID-19, la création des «Fonds spéciaux pour la gestion de la pandémie du coronavirus ", se fait manifester. Ainsi, une mobilisation des organisations non gouvernementales, des gouvernements, des appels à la contribution des entités privées et publiques est très remarquée ${ }^{\{28\}}$. Il s'agit des fonds qui serviraient normalement à améliorer la stratégie de contrôle des infections contre la COVID-19 comprenant la préparation et la distribution du matériel, la formation sur les mesures de prévention des infections, une stratégie de triage, la limitation du trafic à l'hôpital, la réorganisation des services hospitaliers, la préservation de l'environnement propre, etc. ${ }^{29]}$. Au Togo, le gouvernement a mis en place « un programme de revenu universel de solidarité $»\{30\} . \mathrm{Au}$ Burkina Faso, le «Coronathon» mis en place faisait appel à chacune des forces vives du pays à contribuer au fond de riposte ${ }^{\{31}$. La République Démocratique du Congo s'est orientée vers la mobilisation d'un fond de lutte contre la COVID-19'132\}. Bien que ces financements aient donné des résultats satisfaisants dans de nombreux pays, en République Démocratique du Congo, les personnes interrogées fustigent les effets non palpables de ces fonds sur le terrain surtout au regard des nouveaux cas qui restent très croissants. D'aucuns pensent donc que la COVID-19 n'était qu'une stratégie pour se procurer des fonds. Cette représentation devrait être combattue si l'on désire enrayer définitivement la COVDID-19.

\section{COVID-19 : une guerre des états}

La population Kinoise considère la COVID-19 comme une pandémie dont l'origine est les conflits des grandes puissances. Cette considération rejoint celle de Struye et ses collègues ${ }^{\{23\}}$ qui ont montré que :

« la pandémie de la COVID-19 est en train de transformer la configuration géopolitique $\mathrm{du}$ monde et les rapports de force entre les États. L'exemple le plus frappant est celui de la Chine, profitant de la crise de la COVID-19, intensifie son assistance internationale aux pays africains sous diverses formes notamment via l'Éthiopie (respirateurs, équipements de protection, kits de test et masques) au moment où les ÉtatsUnis et l'Europe, qui sont des bailleurs traditionnels des pays africains, gèrent l'expansion rapide de la crise et les décès massifs sur leurs propres territoires. Cette aide chinoise vers l'Afrique est aussi controversée, car elle renforcerait sa présence stratégique sur le continent. "Sur le continent, la santé publique est désormais associée à la nouvelle route de la soie que Pékin cherche à dérouler à travers le monde»\{23\}.

Cette population fuyant le confinement reste très sceptique et considère que la COVID-19 serait une invention pour les conflits de positionnement des grandes puissances. C'est pourquoi Struye considère que " la crise du Covid-19 serait l'équivalent pour Pékin de l'entrée en guerre des États-Unis dans la Première guerre mondiale, premier signe de la puissance américaine qui triomphera après la seconde guerre mondiale $»\{33\}$.

\section{COVID-19 : un châtiment divin}

Le sens accordé à la crise de la COVID-19 en République Démocratique du Congo n'épargne pas le secteur religieux où la COVID-19 prend place de la punition. Cette façon de voir les 
choses pour certains congolais, appuie l'idée de Dujin qui affirme que la pandémie, serait un « avertissement »\{22\}. Loin d'être une apocalypse, les interrogés pensent que la COVID-19 semble prendre la tournure d'annoncer de prochaines crises, forcément plus graves. Toutes ces représentations sociales de la COVID-19 par les Kinois prouvent à suffisance le faible niveau de littératie de cette population en matière de cette pandémie.

\subsubsection{La motivation appuyant la renonce au confinement}

\section{La crise socioéconomique}

Plusieurs personnes interrogées soulignent se déplacer de la ville de Kinshasa vers la province du Kongo Central au regard de la crainte de vivre une véritable crise pendant la période de confinement. Cet argument peut avoir du sens si on s'appuie sur les idées de Zogning considérant qu'en Afrique au moins près de $90 \%$ de la population dépend essentiellement du secteur informel (artisanat, réparation, restauration, petit commerce, etc. ${ }^{\{34\}}$. A ce sujet, Tougan et Théwis, notent que «l'impact négatif de la crise du COVID-19 sur le bien-être des ménages sera considérable »\{35]. Pour les interviewés, le confinement de la ville de Kinshasa entrainerait comme conséquences la pénurie des denrées alimentaires avec comme corollaire la hausse des prix avec des effets désastreux sur le tissu social. En outre, de nombreuses personnes en Afrique ne disposent pas de vivres et dépendent du salaire journalier pour survivre, ce qui les empêche de rester chez elles pendant une période prolongée. Par ailleurs, la capacité du système de santé déjà faible pourrait s'éroder davantage si un grand nombre de travailleurs de la santé tombaient malades ${ }^{\{36\}}$.

\section{Insécurité grandissante}

Les mesures de confinement dans la lutte contre la pandémie de Covid-19 n'a pas que des effets préventifs de cette pandémie. Il a été relevé dans d'autres contextes que pendant cette période, une forte augmentation des cas de cambriolages, d'agressions physiques a été constatée ${ }^{[37]}$. D'autres formes d'insécurité comme la violence familiale et conjugale, la criminalité et les tensions sociales ont aussi été révélées dans d'autres contextes, notamment au sein du domicile, entre les groupes sociaux ${ }^{[38\}}$.

Le Comité en prévention et promotion estimait une augmentation de la criminalité relative à la pandémie dans les mois, voire les années à venir, lorsque les conséquences économiques de la pandémie seront palpables ${ }^{\{39]}$. Cependant, dans le contexte de cette étude, les personnes interrogées soulignent une transposition des cas de la criminalité de la commune de la Gombe au centre de la ville de Kinshasa alors l'épicentre de l'infection à COVID-19 où le confinement était effectif vers les quartiers périphériques. Cela rejoint Marc Metdepenningen ${ }^{\{38\}}$ qui déclare que « le coronavirus contamine aussi la criminalité ». Il semble donc pertinent pour les autorités que la stratégie du confinement s'accompagne de mesures l'encadrement sécuritaire de la population. Nous estimons que lorsque l'insécurité pendant le confinement favorise le déplacement de la population, il y a risque de favoriser la propagation de l'infection à COVID19 de la capitale vers la province.

\section{La recherche du lieu des prières}

Les résultats mettent également en lumière que le confinement ainsi que les autres mesures qui l'accompagnent en l'occurrence la fermeture des lieux de prière a contribué au déplacement de la population. Ce constat rejoint la publication de Schlegel ${ }^{\{40\}}$ qui stipulait que même en Europe où la religion est en chute, dans des contextes tragiques, le besoin impérieux de religion se fait sentir. L'apparition de la COVID-19 touchant directement le spirituel chez les Africains, un renforcement des tendances identitaires et traditionnalistes des religions se fait sentir, etc. Or pour chaque individu, la crise COVID-19 aura modifié le quotidien ${ }^{\{11\}}$. Il semble évident qu'à des pareilles circonstances que des personnes choisissent de se déplacer de Kinshasa vers un autre lieu où ils peuvent trouver des occasions de prier librement. Toutefois, ce déplacement incontrôlé pourrait aussi favoriser la propagation du virus vers les zones non touchées. Une bonne éducation de la population en cette matière semble importante. 


\section{La recherche du traitement traditionnel}

La population interrogée pense qu'avec le confinement annoncé et les frontières fermées, en quittant la capitale pour le milieu rural, les personnes auront la facilité de faire recours aux plantes médicinales nécessaires pour guérir la COVID-19. En effet, en l'absence de tout traitement curatif, les vertus de diverses plantes de la pharmacopée traditionnelle pour soigner la Covid-19 prennent une place importante actuellement. Cela rejoint Doumbia lorsqu'il signale qu'en Afrique en général et particulièrement en République Démocratique du Congo, plus de $80 \%$ de la population ont recours à la médecine traditionnelle en matière de santé ${ }^{\{22\}}$. En effet, depuis la préhistoire, l'être humain recherche dans son environnement (plantes, animaux, pierres, esprits) de quoi soulager ses maux et traiter ses blessures ${ }^{[43]}$. Dans la plupart des pays d'Afrique, les plantes médicinales constituent un arsenal thérapeutique à disposition des guérisseurs traditionnels qui soignent dans certains cas plus de $90 \%$ des maladies ${ }^{[44]}$. Car en milieu rural, les patients et les tradipraticiens évoluent le plus souvent dans un système commun d'interprétation traditionnelle de la maladie. Bien qu'au mieux, ces plantes médicinales peuvent soulager certains symptômes comme la toux ou la fièvre, mais ne prouvent pas encore qu'elles peuvent effectivement soigner ou pas du coronavirus. Faute de preuve scientifique, cette démarche devrait nécessiter une bonne information au regard des effets néfastes sur la santé qu'elles peuvent causer.

\subsubsection{Artefacts thérapeutiques pour se soigner la COVID-19}

Les propos recueillis de personnes interrogées approuvent qu'ils utilisent des stratégies pour une éventuelle prévention et guérison des personnes atteintes de la COVID-19. Il s'agit d'une médecine traditionnelle considérée ici comme artefacts dans la mesure où certains effets attribués à celle-ci ne sont que psychologiques, non prouvés scientifiquement [42\}. Selon les interviewés de cette étude, leurs approches thérapeutiques sont faites des composés d'herboristes et des croyances traditionnelles.

\section{Des herboristes}

Le Vernonia amygdalina est la première plante la plus citée par les enquêtés. Il s'agit d'une plante très populaire dans le continent africain. Selon Erasto, Grierson et Afolayan ${ }^{\{45}$. Elle est retrouvée en en République Démocratique du Congo, Guinée, en côte d'Ivoire, au Mali, en Sierra Leone, au Togo, au Nigeria, en Ouganda, au Congo Brazzaville, au Kenya, en Tanzanie, etc.. En République Démocratique du Congo, le Vernonia amygdalina est connu dans la capitale sous le nom de «Kongo Bololo» ou «Malulu »en Lingala. Il est aussi appelé Kilulukunju en Swahili; mandudindudi en Kikongo; (Kongo bololo). C'est une plante à laquelle on reconnait plusieurs usages médicinaux généralement à cause de son goût très amère. Pour cette raison, elle est utilisée dans le traitement du paludisme, des hépatites, des vers intestinaux ${ }^{\{45\}}$. Parfois aussi, elle joue un grand rôle dans la guérison des infections cutanées ${ }^{[46)}$. En raison de son utilisation dans la lutte contre la malaria ${ }^{[47\}}$, la population souligne l'avoir simulée à la chloroquine au moment où cette molécule suscitait des débats dans le traitement de la COVID-19. Fonder les espoirs sur cette plante qui a eu des grands succès et dont " plusieurs vendeurs ambulants ont inondé les rues de Kinshasa, vantant les vertus médicinales de cette plante face au coronavirus ${ }^{(46)}, c^{\prime}$ est désorienter tout un peuple de bonnes stratégies d'élimination de la COVID-19. De grands efforts de sensibilisations sont donc souhaités.

\section{La fumigation traditionnelle}

La fumigation traditionnelle est une autre issue thérapeutique contre la COVID-19 soulignée par les sujets de l'étude. Cela rejoint Anne Peron qui affirmait que depuis les peuples traditionnels, la fumigation était considérée comme méthode primitive de purification de l'atmosphère souvent teintée d'aspects énergétiques et religieux ${ }^{[48)}$. D'ailleurs, une étude indienne mettant en lumière les possibilités de la fumigation, a rapporté qu'une heure de fumigation par combustion d'un mélange de bois et d'herbes médicinales et odoriférantes entraînait une réduction de 94 \% de la population bactérienne aérienne ${ }^{\{49\}}$. Bien 
que ces effets soient présumés, une bonne information de la population serait toujours nécessaire afin que celle-ci ne bannisse point les mesures barrières contre la COVID-19, en considérant la fumigation comme méthode préventive et curative par excellence.

\section{Les croyances traditionnelles}

Les prières religieuses

Le recours à des prières religieuses est une forme thérapeutique très répandue en Afrique pour faire face à des catastrophes sanitaires. À ce sujet Mabakutuvangilanga Ntela a considéré dans son étude qu'en matière de santé, les messages des églises créent une véritable dissonance cognitive en milieu rural de la République Démocratique du Congo ${ }^{\{50\}}$. À ce titre, ces messages constituent à la fois une opportunité et une menace. Cette voie peut être une opportunité dans la mesure où, intégrées au sein des communautés, OMS souligne que les autorités religieuses constituent ${ }^{\{51\}}$, pour les communautés au service desquelles elles œuvrent, une source essentielle de soutien, de réconfort, de conseils, de services sociaux et de soins de santé directs. Dans ce cadre, elles peuvent jouer un rôle important dans la réduction de la morbidité liée à la COVID-19 notamment dans la diffusion des informations sanitaires visant à protéger leurs membres. En effet, les adeptes sont plus susceptibles d'accepter des informations provenant de leurs encadreurs spirituels que celles d'autres sources. Cependant, spiritualisme peut aussi être une menace pour la santé lorsque certaines églises prônent la guérison miraculeuse à travers les prières. Au Burundi, l'ex président a instruit à ses administrés que son pays était protégé par Dieu, bafouant donc les mesures barrières à la COVID-19 pour laquelle sa propre femme en a été victime COVID-19. À ce sujet, un des émissaires de pays déclamait: "Le Burundi est une exception car c'est un pays qui a donné à Dieu la première place »\{52\}. Cette dissonance s'est faite remarquée récemment en Tanzanie, pendant que le président était inflexible sur les jeûnes et prières ayant réduit selon lui le nombre des malades de la COVID19 dans les hôpitaux, refusant ainsi de publier les statistiques quotidiennes telles qu'elles étaient réclamées par l'opposition ${ }^{\{52\}}$. De son côté, l'ambassade américaine avait mis en garde dans un communiqué contre le risque "très élevé" de contamination à Dar es Salaam, invitant son personnel et leur famille à rester chez eux et fustigeant le débordement des hôpitaux faisant état de signes de "croissance exponentielle" du virus dans la ville et d'autres endroits du pays ${ }^{\{33\}}$.

\section{Le fétichisme et marabouts}

Le recours au fétichisme et aux marabouts est la solution thérapeutique la moins soulevée par les sujets. Cela corrobore Matteo Maillard qui stipule en effet que, pendant qu'il n'existe jusque-là aucun traitement curatif contre la COVID-19 et dont les mesures barrières constituent le meilleur moyen de la prévenir, dans le continent africain, des féticheurs, des marabouts... et autres formes des charlatans désorientent la population vers des remèdes possibles des "guérisseurs" contre le coronavirus ${ }^{\text {\{54. }}$. L'aute- $\underline{\text { un }}$ explicite qu'au Bénin, au Sénégal ou au Mali, marabouts et tradipraticiens proposent des solutions qui relèvent, au choix, de la médecine douce ou du charlatanisme. Au regard de l'ampleur de la COVID-19, des solutions thérapeutiques non prouvées scientifiquement considérées dans cette étude comme des artefacts thérapeutiques que l'Afrique se propose, il y a lieu de justifier à suffisance la place combien importante des stratégies éducationnelles adaptées au contexte.

\subsubsection{Conséquences de ces représentations} L'effet du boomerang

A l'examen de ces quelques représentations sociales de la COVID-19 dont la liste n'est sûrement pas exhaustive, on ne peut se soustraire à l'évidence du caractère symétrique des effets boomerang qui s'exercent sur la population. Jarry-Omarova considère l'effet boomerang comme "le résultat soudain obtenu en réaction à la réception d'une communication et dont les caractéristiques sont le plus souvent inattendues et négatives ${ }^{\{55 \mid}$. Les différents discours sur la COVID-19 ne sont donc pas tous au bénéfice de l'adoption d'un 
comportement préventif pour la population. Bien au contraire, certains discours soulignés font que la cible visée soit manquée ». Des tels résultats impliquent que des actions à mettre en place ne devraient pas être copiées de l'extérieur, mais devraient plutôt prêter attention à l'ensemble de la situation sociopolitique globale, aux spécificités culturelles de différentes communautés, à la vision du futur et les perspectives de projets sociaux.

La réactance psychologique des individus

La COVID-19 en général et particulièrement l'annonce du confinement est un évènement nouveau qui a suscité de la réactance psychologique au sein de la population Kinoise. Il s'agit bien « d'un état émotionnel, un sentiment souvent désagréable qui émerge lorsque l'on ressent une menace ou une entrave à la liberté de choix ${ }^{[56)}$. De ce fait, l'humain vit dans une nécessité de se sentir libre, de s'engager dans certains comportements et d'être à l'initiative de ses différentes actions ». Dans le cas d'espèce, la réactance psychologique entre en jeu lorsque les individus ont considéré le confinement comme une privation de leur liberté. Cela a entrainé chez certaines personnes une forme d'inconfort psychologique, chez d'autres un sentiment désagréable motivant leur état fugitif. A ce sujet, Romain fait remarquer que "dans des contextes sanitaires, la théorie de la réactance psychologique offre un cadre pour préparer les campagnes de communication à l'échelle de la population afin d'améliorer leur impact »[57]. Cela justifie à suffisance la place de la bonne communication sans laquelle, des individus sont susceptibles de considérer les mesures barrières de la COVID-19 comme des tentatives de manipulation de leur comportement pouvant se traduire par un abandon ou un rejet à l'observance des celles-ci.

\subsubsection{Intérêt $d^{\prime} u n e$ éducation approfondie sur la COVID-19}

Toutes les formes de considération attribuées à cette pandémie telles qu'elles sont démontrées dans cette étude, constituent un véritable obstacle à la lutte contre la COVID-19 qui prend pourtant une allure inquiétante en Afrique. Cela montre un besoin impérieux d'amélioration des stratégies de communication actuelle. Il s'agit alors de proposer une communication qui tient compte non seulement de la transmission du message, mais aussi de sa compréhension et surtout de son utilisation par les usagers ${ }^{1587}$. Parmi les éléments à prendra en compte : le niveau de littératie en santé des personnes, c'est-à-dire la motivation et les compétences des individus à accéder, comprendre, évaluer et utiliser l'information en vue de prendre des décisions appropriées concernant leur santé.

Nous proposons ainsi une communication qui remet en cause la communication médiatique actuelle, à double étage, qui privilégie la force des relations interpersonnelles et met en avant l'intervention de leaders d'opinion avant de toucher la population cible. Pour cela, il s'agit de mettre en place des stratégies de communication qui intègrent aux connaissances médicales des spécificités locales et culturelles ${ }^{\{59}$. La prise en compte de ces spécificités peut faciliter la compréhension des logiques internes des catégories, des valeurs et des pratiques largement répandues au sein des populations et d'en favoriser l'acceptation et l'appropriation ${ }^{\{60\}}$.

Par ailleurs, la communication ne suffira pas pour obtenir des changements profonds de comportements de santé face à l'épidémie. Par conséquent, il semble également indispensable de proposer une éducation pour la santé, dans la communauté, en tenant compte de l'environnement des personnes et notamment des plus vulnérables ${ }^{\{61\}}$. Cette " éducation d'urgence ", telle que proposée par le Laboratoire Educations et Pratiques de Santé (LEPS UR 3412) de l'Université Sorbonne Paris Nord, couplée à une stratégie d'information, vise à l'appropriation de compétences en santé nécessaires pour répondre à la crise sanitaire actuelle ${ }^{\{61\}}$.

\subsubsection{Limites et considérations méthodologiques}

Les résultats de cette étude exploratoire doivent être interprétés à la lumière de ses limites liées aux caractéristiques des participants, aux choix méthodologiques et à la réalisation des entretiens. Il semble important 
que des études similaires soient menées dans d'autres contextes afin d'enrichir les résultats, lesquels résultats peuvent déjà orienter les interventions à venir sur la COVID-19 dans ce contexte.

\section{Conclusion}

Les résultats de cette étude ont permis l'exploration des représentations sociales qu'ont les personnes ayant quitté la ville de Kinshasa à l'annonce du confinement dans le cadre de la lutte contre la COVID-19, de comprendre les raisons de pouvoir quitter la ville ainsi que les solutions thérapeutiques considérées comme des «artefacts thérapeutiques» parce qu'estimées comme imaginaires. La démarche de recherche qualitative déployée pour cette étude et l'hétérogénéité relative des caractéristiques des répondants et de leurs expériences personnelles a enrichi la lecture du phénomène à l'étude par la considération de différents aspects qui leur sont propres.

D'une part, les résultats de l'étude présentés dans cet article mettent en lumière l'importance fondamentale de renforcer davantage l'intérêt à accorder au suivi des populations déplacées dans le contexte de la COVID-19. D'autre part, ces résultats montrent le grand besoin

\section{Références}

\{1\}. WHO. (2020). Communication sur les risques et participation communautaire (RCCE) : Préparation et riposte face au nouveau coronavirus 2019 (2019-nCoV): Lignes directrices provisoires.

https://apps.who.int/iris/bitstream/handle/10665/33 0679/9789240000797-fre.pdf

\{2\}. Yapi-Diahou, A. (2020). Cahier «COVID 19 au quotidien » (p. 125 p.).

https://hal-univ-paris8.archives-ouvertes.fr/hal$\underline{02859844}$

\{3\}. Guan, W., Ni, Z., Hu, Y., Liang, W., Ou, C., He, J., Liu, L., Shan, H., Lei, C., Hui, D. S. C., Du, B., Li, L., Zeng, G., Yuen, K.-Y., Chen, R., Tang, C., Wang, T., d'informer suffisamment la population congolaise sur la COVID-19 qui continue jusque-là gagner du terrain, dans la mesure où une représentation sociale n'est pas un savoir théorique objectif. Elle est plutôt une prise de position, un jugement sur la nature et la portée, lequel jugement n'est pas toujours tout à fait conforme à la « réalité ». Or, en raison même du caractère situé et construit de toute représentation sociale qui suppose qu'elle est « redevable aux pratiques et aux croyances ambiantes $\aleph^{\{62\}}$, l'existence d'un décalage entre l'objet représenté et sa représentation est inévitable.

Dans la perspective d'approfondir le sujet de cette étude, il serait intéressant de réaliser des analyses plus poussées, guidées par un devis de recherche mixte, en sollicitant un échantillon plus important. La désinformation et les rumeurs généralisées au départ de la COVID19 ayant alimenté la méfiance à l'égard des institutions formelles et ont amené les communautés à résister à agir de manière critique pour leur propre sécurité, l'adaptation de la riposte à l'épidémie au contexte local en impliquant les leaders d'opinion de chaque population concernée est une nécessité.

Conflit d'intérêt : Aucun conflit d'intérêt n'a été constaté.

Chen, P., Xiang, J., ... Zhong, N. (2020). Clinical Characteristics of Coronavirus Disease 2019 in China. New England Journal of Medicine, 0(0), null. https://doi.org/10.1056/NEJMoa2002032

\{4\}. Martin, P. (2015). Contraintes vécues, idéal normatif et actions déployées en vue de transformer l'exercice de la profession infirmière en centre hospitalier: Une étude exploratoire auprès d'infirmières québécoises politiquement engagées. https://papyrus.bib.umontreal.ca/xmlui/handle/186 $\underline{6 / 13042}$ 
\{5\}. Greenstone, M., \& Nigam, V. (2020). Does Social Distancing Matter? (SSRN Scholarly Paper ID 3561244). Social Science Research Network. https://doi.org/10.2139/ssrn.3561244

\{6\}. Ecol'Aube, F. (2020). Festival Ecol'Aube: Le covid-19, un drame pour sauver le monde? - [Yonne Lautre]. http://yonnelautre.fr/spip.php?article5737

\{7\}. Xiao, Y., \& Torok, M. E. (2020). Taking the right measures to control COVID-19. The Lancet Infectious Diseases, 20(5), 523-524.

https://doi.org/10.1016/S1473-3099(20)30152-3

\{8\}. Moliner, P. (2008). Représentations sociales et iconographie. Communication et organisation. Revue scientifique francophone en Communication organisationnelle, 34, 12-23.

https://doi.org/10.4000/communicationorganisation. $\underline{547}$

\{9\}. Herzlich, C. (2019). Santé et maladie. Analyse d'une représentation sociale. Walter de Gruyter $\mathrm{GmbH} \& \mathrm{Co}$ KG.

\{10\}.Nappa, J., Schoumaker, B., Phongi, A., \& Flahaux, M.-L. (2019). Difficultés économiques et transformation des unions à Kinshasa. Population, Vol. 74(3), 273-298.

11. Wubneh, M. (1991). Review of African Cities in Crisis : Managing Rapid Urban Growth [Review of Review of African Cities in Crisis: Managing Rapid Urban Growth, par R. E. Stren \& R. R. White]. African Studies Review, 34(3), 151-153.

https://doi.org/10.2307/524151

\{12\}.De Boeck, F. (2020). Urban expansion, the politics of land, and occupation as infrastructure in Kinshasa. Land Use Policy, 93, 103880. https://doi.org/10.1016/j.landusepol.2019.02.039.

$\{13\}$.Lin, C.-Y. (2020). Social reaction toward the 2019 novel coronavirus (COVID-19). Social Health and Behavior, 3(1), 1. https://doi.org/10.4103/SHB.SHB 1120

\{14\}.Bachelor, A., \& Joshi, P. (1986). La méthode phénoménologique de recherche en psychologie: Guide pratique. Presses Université Laval.

\{15\}.Alvaro Pires. (1997). "Échantillonnage et recherche qualitative: Essai théorique et méthodologique". Criminologue, École de criminologie, Université d'Ottawa.

http://classiques.uqac.ca/contemporains/pires alvar o/echantillonnage recherche qualitative/echantillo n recherche qual.pdf?

\{16\}.L'Écuyer, R. (2011). Méthodologie de l'analyse développementale de contenu. PUQ.
\{17\}.Negura, L. (2006). L'analyse de contenu dans l'étude des représentations sociales. SociologieS. http://journals.openedition.org/sociologies/993

\{18\}.Migabo, V. (2020). L'Afrique face à la Covid-19: Une riposte inégale. Université du Québec à Montréal (UQAM) provides funding as a founding partner of The Conversation CA-FR.

https://theconversation.com/lafrique-face-a-lacovid-19-une-riposte-inegale-136896

\{19\}.Hamon-Valanchon, H. (2009). Femmes et cancer : Imaginaire de la maladie et culture hospitalière. Societes, $n^{\circ} 105(3), 57-69$.

\{20\}.Moulin, P. (2005). Imaginaire social et Cancer. Revue Francophone de Psycho-Oncologie, 4(4), 261-267. https://doi.org/10.1007/s10332-005-0094-y

\{21\}.Augustin Voegele, A. (2018). "Knock, ou les bien portants imaginaires ».

https://halshs.archives-ouvertes.fr/halshs-02086128

\{22\}.Dujin, A. (2020). Le virus dans la cité. Esprit, Mai(5), $37-41$.

\{23\}. Rey, L., \& Dare, M. (2020). La gestion de la pandemie du coronavirus en context African : Quells enjeux? 2020, 18.

\{24\}.Cathébras, P. (1997). Qu'est-ce qu'une maladie? La Revue de Médecine Interne, 18(10), 809-813. https://doi.org/10.1016/S0248-8663(97)89972-9

\{25\}.Dumont, A. (2017). Réduire la mortalité maternelle dans les pays en développement: Quelles sont les interventions efficaces? Revue de médecine périnatale, 9(7), 14.

\{26\}.Deluzarche, C. (2021). Les grandes pandémies qui ont marqué l'histoire. Futura.

https://www.futura-sciences.com/sciences/ questions-reponses/histoire-grandes-pande miesont-marque-histoire-13440/

\{27\}.Peter St. Onge. (2020). Pour un stimulus fédéral ciblé contre le coronavirus. LE POINT, 2.

\{28\}.Abdelaaziz Ait Ali, Abdelhak Bassou, M'hammed Dryef, Karim El Aynaoui, Rachid El Houdaigui, Youssef El Jai, Faiçal Hossaini, Larabi Jaidi, Mohamed Loulichki, \& El Mostafa Rezrazi, Abdallah Saaf. (2020). La stratégie du Maroc face au covid-19. Policy Center for the New South. https://www.policycenter.ma/sites/default/files/PP20-07 La strategie du Maroc Face Au Covid19.pdf

\{29\}.Lu, Wang, Yu, Yang, Zhao, D., Y, H, Y. (2020). Integrated infection control strategy to minimize nosocomial infection of coronavirus disease 2019 
among ENT healthcare workers. The journal of hopital infection, Elsevier.

https://doi.org/10.1016/j.jhin.2020.02.018

\{30\}.Rouguyata Sall. (2020). Au Togo, le coronavirus a accéléré la mise en place d'un revenu universel de solidarité.

https://www.lemonde.fr/afrique/article/2020/10/23/a u-togo-le-coronavirus-a-accelere-la-mise-en-place-

d-un-revenu-universel-desolidarite 6057162 3212.html

\{31\}.Kahofi Jischvi SUY. (2020). Coronavirus en Afrique : La lutte contre le Covid-19 avec des moyens africains. News Afrique.

https://www.bbc.com/afrique/region-52608574

\{32\}.Le bulletin d'actualité économique de la RDC. (2020). COVID-19: Impacts et perspectives sur l'économie de la R.D. Congo. BELTRADE, 49. file:///C:/Users/Prof\%20Decap/Downloads/BeltradeInfo-n-49-version-web.pdf

\{33\}.Struye, T., Desplanque, S., Liégeois, M., Orinx, K., Rosoux, V., \& Vandamme, D. (2020). Relations internationales: Quelques leçons. CECRI, 9.

\{34\}.Zogning, F., Mbaye, A. A., \& Um-Ngouem, M.-T. (2017). L'économie informelle, l'entrepreneuriat et l'emploi. Editions JFD

\{35\}.Tougan U.P, Théwis A. (2020). [Covid-19 and Food Security in Sub-Saharan Africa: Implications and Proactive Measures to Mitigate the Risks of Malnutrition and Famine]. International Journal of Progressive Sciences and Technologies (IJPSAT), 20(1), 172-193.

\{36\}.Patrick Dupoux , Jim Larson, Shalini Unnikrishnan. (2020). Fighting COVID-19 in Africa Will Be Different.

https://www.bcg.com/publications/2020/fightingcovid-in-africa.aspx.

\{37\}.The Guardian. (2020). Criminalité.En Afrique, le Covid-19 favorise l'insécurité. Courriel international. https://www.courrierinternational.com/revue-depresse/criminalite-en-afrique-le-covid-19-favoriselinsecurite

\{38\}.Marc Metdepenningen. (2020). La criminalité infectée par le coronavirus. Le soir. https://plus.lesoir.be/293767/article/2020-04-10/lacriminalite-infectee-par-le-coronavirus

\{39\}.Comité en prévention et promotion. (2020). COVID19-Exploration des indicateurs de suivi de la violence, de la sécurité, du sentiment de sécurité, de la criminalité et des tensions sociales. Institut National de Santé Publique du Québec.

https://www.inspq.qc.ca/sites/default/files/covid/29 85-suivis-violence-securite-criminalite-tensionssociales-covid19.pdf

\{40\}.Schlegel, J.-L. (2020). La religion au temps du coronavirus. Esprit, Mai(5), 69-76.

\{41\}.Faure, A. (2020). «La crise du Covid vue par le monde de la recherche »: Le virus de la recherche. Presses universitaires de Grenoble, Volet I.

http://journals.openedition.org/lectures/41676

\{42\}.Doumbia, H. (2015). Place de la médecine traditionnelle dans la prise en charge thérapeutique des enfants de moins de 5 ans avant leur hospitalisation au CSRéf de Koutiala. https://www.bibliosante.ml/handle/123456789/741

\{43\}.Sofowora, A. (2010). Plantes médicinales et médecine traditionnelle d'Afrique. KARTHALA Editions.

\{44\}.Abayomi, S. (2010). Plantes médicinales et médecine traditionnelle d'Afrique. Nouvelle édition. KARTHALA Editions.

\{45\}.Erasto P, Grierson DS, Afolayan AJ. Bioactive sesquiterpene lactones from the leaves of Vernonia amygdalina. J Ethnopharmacol. 2006 Jun 15;106(1):117-20. doi: 10.1016/j.jep.2005.12.016. Epub 2006 Feb 3. PMID: 16458461.

\{46\}.Digital Congo. (2020). Coronavirus à Kinshasa: Des escrocs tentent de jouir de la crise. Coronavirus en $R D C$.

https://www.digitalcongo.net/article/5e7cd3d8c46fc $\underline{30004 d f 5218 /}$

\{47\}.Abosi, A. O., \& Raseroka, B. H. (2003). In vivo antimalarial activity of Vernonia amygdalina. British Journal of Biomedical Science, 60(2), 89-91. https://doi.org/10.1080/09674845.2003.11783680

\{48\}.Anne Peron. (2017). Bains, cataplasmes, massages... Faites vos soins maison. Plantes et Sante : Le site de la phytotherapie. https://www.plantes-etsante.fr/articles/phytotherapie/3292-fumigationsinhalations-et-olfactotherapie

\{49\}.Jean-Pierre Giess. (2017). La fumigation: Traditions et bonnes pratiques. PLANTES \& SANTÉ : LE SITE DE LA PHYTOTHÉRAPIE. https://www.plantes-etsante.fr/articles/conseils-dutilisation/2428-lafumigation-traditions-et-bonnes-pratiques

\{50\}.Ntela, S.-D. M., Goutte, N., Morvillers, J.-M., Crozet, C., Ahouah, M., Omanyondo-Ohambe, M.-C., NtotoKunzi, B., Kandolo, F. T., \& Rothan-Tondeur, M. (2018). Observance to antiretroviral treatment in the 
rural region of the Democratic Republic of Congo : A cognitive dissonance. The Pan African Medical Journal, 31.

https://doi.org/10.11604/pamj.2018.31.159. 15132

\{51\}.OMS. (2020). Considérations pratiques et recommandations à l'intention des autorités religieuses et des communautés de croyants dans le contexte de la COVID-19 Orientations provisoires. OMS

https://apps.who.int/iris/bitstream/handle/10665/33 2046/WHO-2019-nCoV-Religious Leaders-2020.1fre.pdf

\{52\}.Jean-Claude Karerwa Ndenzako. (2020). COVID-19 / "Le Burundi est une exception car c'est un pays qui a donné à Dieu la première place » - IWACU. https://www.iwacu-burundi.org/covid-19-leburundi-est-une-exception-car-cest-un-pays-qui-adonne-a-dieu-la-premiere-place/

\{53\}.AFP. (2020). L'opposition tanzanienne réclame la "transparence» et des bilans du coronavirus-Africa Radio. DÉPÊCHES AFP.

https://www.africaradio.com/news/l-oppositiontanzanienne-reclame-la-transparence-et-des-bilansdu-coronavirus-167700

\{54\}.Matteo Maillard. (2020). «Ce n'est pas difficile à soigner»: En Afrique, les remèdes des «guérisseurs» contre le coronavirus. Le monde. https://www.lemonde.fr/afrique/article/2020/04/11/c e-n-est-pas-difficile-a-soigner-en-afrique-lesremedes-des-guerisseurs-contre-lecoronavirus 6036289 3212.html

\{55\}.Jarry-Omarova, A. (2011). Mouvement associatif des femmes en Mongolie et partenariats internationaux. Exemple d'un « contre-effet boomerang ». Cultures $\mathcal{E}$ Conflits, 83, 79-98. https://doi.org/10.4000/conflits.18201

\{56\}.Mugny, G., \& Papastamou, S. (2009). « Réactance psychologique » et ordre social. Quaderns de Psicologia, 0(8), 103-114. https://doi.org/10.5565/rev/qpsicologia.378

\{57\}.Romain, A. J. (2020). Communication à la population en temps de pandémie: Quintessesnce, 14(S2), 2.

\{58\}.Vigneron, C., Gaudy-Marqueste, C., Dalmazzone, I., Sirvent, A., Guyader, V., Loundou, A., Richard, M.A., Grob, J.-J., \& Mallet, S. (2018). Impact de la littératie sur la corticophobie et la prise en charge de la dermatite atopique. Annales de Dermatologie et de Vénéréologie, 145(12, Supplement), S60-S61. https://doi.org/10.1016/j.annder.2018.09.027.
\{59\}.Richard, C., Lussier, M.-T., Galarneau, S., \& Jamoulle, O. (2010). Compétence en communication professionnelle en santé. Pédagogie Médicale, 11(4), 255-272.

https://doi.org/10.1051/pmed/2011009

$\{60\}$.Schwartz, S. H. (2006). Les valeurs de base de la personne: Théorie, mesures et applications. Revue francaise de sociologie, Vol. 47(4), 929-968.

\{61\}.Margat, A., Pétré, B., d'Ivernois, J.-F., Lombrail, P., Cailhol, J., \& Gagnayre, R. (2020). COVID-19: Proposition d'un modèle d'éducation d'urgence. Education Thérapeutique du Patient - Therapeutic Patient Education, 12(1), 10402. https://doi.org/10.1051/tpe/2020003

\{62\}.Larochelle, M., \& Désautels, J. (2001). Les enjeux socioéthiques des désaccords entre scientifiques : Un aperçu de la construction discursive d'étudiants et étudiantes. Canadian Journal of Science, Mathematics and Technology Education, 1(1), 39-60. https://doi.org/10.1080/14926150109556450 